Case Report

\title{
Metastatic Cerebellar Gastrointestinal Stromal Tumor with Obstructive Hydrocephalus Arising from the Small Intestine: A Case Report and Review of the Literature
}

\author{
Kunitomo Sato, ${ }^{1}$ Toshihide Tanaka, ${ }^{2}$ Naoki Kato, ${ }^{1}$ \\ Takuya Ishii, ${ }^{1}$ Toru Terao, ${ }^{1}$ and Yuichi Murayama ${ }^{3}$ \\ ${ }^{1}$ Department of Neurosurgery, Atsugi City Hospital, 1-16-36 Mizuhiki, Atsugi city, Kanagawa 243-0004, Japan \\ ${ }^{2}$ Department of Neurosurgery, Jikei University School of Medicine, Kashiwa Hospital, 163-1 Kashiwashita, \\ Kashiwa, Chiba 277-8567, Japan \\ ${ }^{3}$ Department of Neurosurgery, Jikei University School of Medicine, 3-25-8 Nishi-Shinbashi, Minato-ku, Tokyo 105-8461, Japan \\ Correspondence should be addressed to Toshihide Tanaka; ttanaka@jikei.ac.jp
}

Received 14 November 2014; Revised 9 December 2014; Accepted 9 December 2014; Published 28 December 2014

Academic Editor: Raffaele Palmirotta

Copyright (C) 2014 Kunitomo Sato et al. This is an open access article distributed under the Creative Commons Attribution License, which permits unrestricted use, distribution, and reproduction in any medium, provided the original work is properly cited.

\begin{abstract}
Gastrointestinal stromal tumor (GIST) is defined as a c-kit-positive gastrointestinal, mesenteric, or omental mesenchymal tumor that very rarely metastasizes to the brain. Metastasis to the cerebellum is particularly rare. An 80 -year-old man presented with nausea and vomiting with disturbance of consciousness. Magnetic resonance imaging (MRI) revealed tumor in the cerebellar vermis causing obstructive hydrocephalus. The patient subsequently underwent midline suboccipital craniotomy, and the tumor was totally removed. Immunohistochemical analysis showed tumor cells positive for c-kit and CD34, and cerebellar metastasis of GIST was diagnosed. Postoperative radiotherapy was administered. Following surgery and radiotherapy, the patient developed ileus caused by tumor in the small intestine and underwent laparotomy for tumor removal. Following abdominal surgery, left hemiparesis and consciousness disturbance were noted. Computed tomography showed recurrent large tumor with perifocal edema in the right frontal lobe of the brain. The patient died 3 months after initial craniotomy. Intracranial metastasis of GIST is extremely rare. In cases such as the present, where the condition of the patient rapidly deteriorates and features such as rising intracranial pressure and ileus prevent the use of oral agents, molecular-targeted agents administered by intravenous infusion should be utilized.
\end{abstract}

\section{Introduction}

Gastrointestinal stromal tumor (GIST) is a mesenchymal tumor that arises from precursors of the interstitial tissue cells of Cajal in the gastrointestinal tract expressing c-kit and CD34 [1-3].

GIST usually metastasizes to the lungs and liver as a result of hematogenous spread and peritoneal seeding, and metastasis of GIST to the central nervous system (CNS) is extremely rare, with a dismal life prognosis. Most intracranial metastases from GIST have been located in cortical and subcortical areas with relatively easy surgical access and have usually been treated surgically followed by adjuvant therapy such as radiotherapy or chemotherapy.
The development of molecular-targeted anticancer agents is giving new hope to these patients. Imatinib mesylate is the first effective systemic therapy for locally unresectable or metastatic GIST [4]. Since being approved for use in treatment, imatinib mesylate has dramatically improved outcomes for patients with advanced GIST [5].

However, imatinib mesylate is believed to be ineffective against brain metastases, because it cannot pass through the blood-brain barrier (BBB) and does not achieve adequate levels in the brain when administered in lower concentrations [6]. As a second-line treatment, sunitinib, a multitargeted small-molecule tyrosine kinase inhibitor, has been shown to be effective and is currently approved worldwide for metastatic GIST $[7,8]$. 


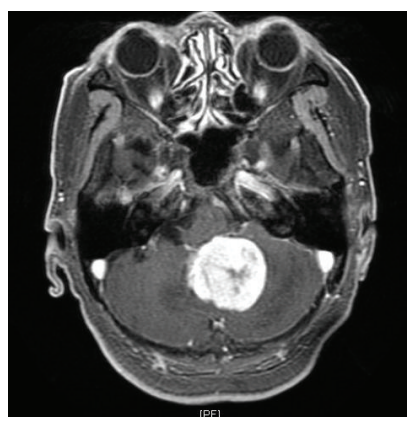

(a)

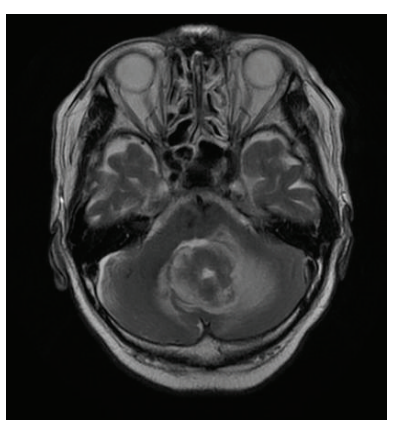

(b)

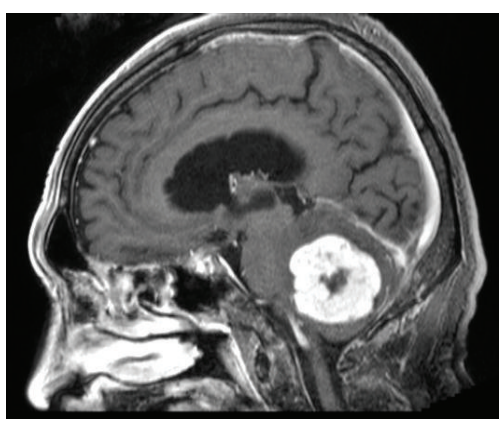

(c)

FIGURE 1: T1-weighted ( $\mathrm{a}, \mathrm{b})$ and T2-weighted (c) magnetic resonance imaging (MRI) revealing the tumor in the vermis with homogeneous enhancement by gadolinium. Note that the tumor compressing medulla resulting in obstructive hydrocephalus (b).

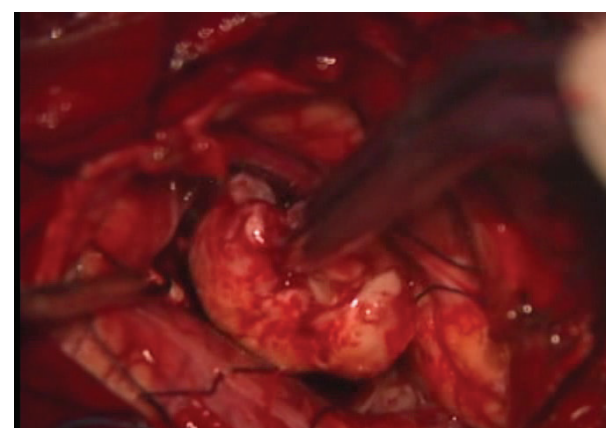

FIGURE 2: Intraoperative findings. The tumor in the vermis was totally removed. The mass appears yellowish, elastic, and hard with a clear boundary from normal structures.

The effectiveness of radiotherapy for GISTs has not been confirmed. GISTs are considered "radioresistant" tumors due to their histological relationship to soft tissue sarcomas, which show relatively poor responsiveness to radiotherapy. Some case reports have indicated that radiotherapy can reduce the tumor burden and produce durable local control for locally advanced and metastatic tumors [9-11]. Radiotherapy can provide both objective responses and symptomatic relief without detracting dramatically from quality of life and should be considered in the multidisciplinary care of patients with locally advanced or metastatic GIST.

Here, we report the case of a patient with cerebellar metastatic GIST that was treated by surgical resection and radiation. In addition, we summarize and review the relevant literature regarding intracranial metastasis of GIST.

\section{Case Report}

The patient was an 80-year-old man who presented with nausea, anorexia, and gait disturbance. Neurological examination revealed dysmetria and dysdiadochokinesia on the left extremities. Magnetic resonance imaging (MRI) showed a round, 4-cm tumor appearing isointense on T1- and T2weighted imaging with homogeneous enhancement (Figures $1(\mathrm{a})-1(\mathrm{c})$ ) causing obstructive hydrocephalus. Since his condition was progressively deteriorating with consciousness disturbance rapidly, midline suboccipital craniotomy was subsequently performed to remove the tumor. The tumor was elastic, hard, and yellowish and displayed clear separation from the pia of the medulla oblongata. The tumor was totally removed (Figure 2).

Histological examination showed spindle-shaped cells with round nuclei and many mitotic figures. Spindle-shaped cells with moderate cellularity were crowded into bunches with mitotic figures (Figure 3(a)). Based on immunohistochemical studies revealing positivity for c-kit, CD34, and $\alpha$ smooth muscle actin (Figures 3(b)-3(d)), the patient was diagnosed with GIST. At that time, no tumors were detected on endoscopic investigation of the gastrointestinal tract.

Postoperatively, the patient showed an improved level of consciousness and postoperative MRI showed no residual tumors (Figure 4(a)). Following craniotomy, endoscopic examination of the gastrointestine and colon showed no abnormal findings except atrophic gastritis. One month after craniotomy, radiotherapy was commenced as postoperative adjuvant therapy. Unfortunately, the patient suffered from severe abdominal pain and vomiting, which turned out to be ileus during radiotherapy. Positron emission tomography (PET) revealed multiple accumulations in the cardiac apex, subclavial region, and peritoneal cavity (Figure 4(b)). Radiotherapy was suspended (final total, 22 Gy in 11 fractions), and laparotomy was subsequently undertaken to achieve relief from the ileus with removal of the small intestinal tumor, which was identified as the primary GIST lesion. Systemic treatment with imatinib mesylate was not administered because of the patient's systemic condition. Three weeks after removal of the small intestinal tumor, the patient suffered from disturbance of consciousness with left hemiparesis. CT showed multiple recurrent tumors in the right frontal lobe with expanding perifocal edema (Figure 5). The condition of the patient gradually deteriorated and he died 4 months after initial craniotomy. Agreement for autopsy was not obtained.

\section{Discussion}

GIST mostly metastasizes within the abdomen, and the most common sites for the development of metastasis are the liver 


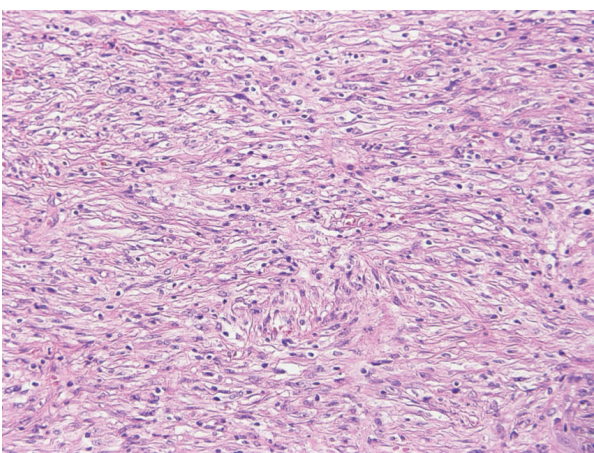

(a)

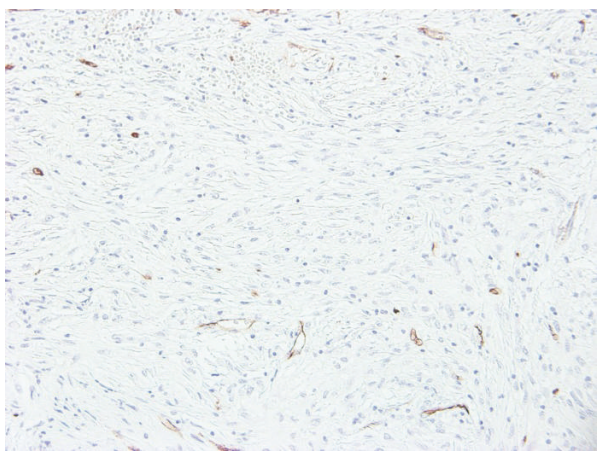

(c)

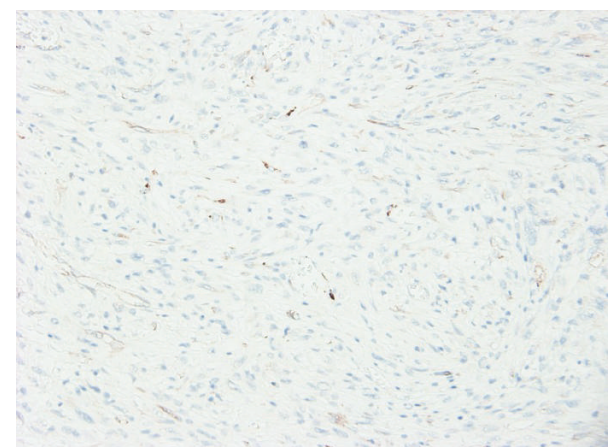

(b)

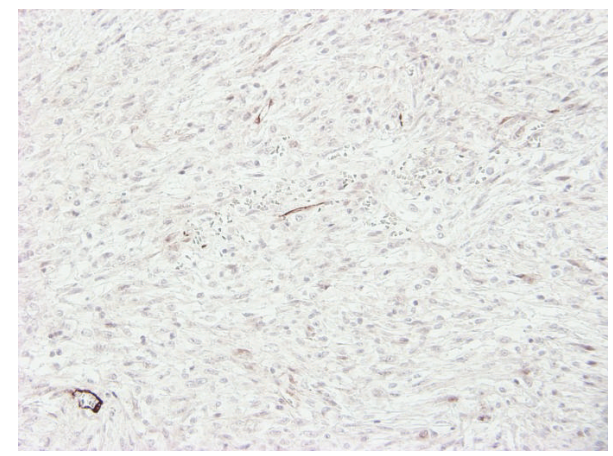

(d)

FIGURE 3: Microscopic photograph showing spindle-shaped cells with moderate cellularity crowded into bundles (hematoxylin and eosin stain, $\times 20)(a)$. Immunohistochemical studies reveal that tumor cells are positive for c-kit (b), CD34 (c), and $\alpha$-smooth muscle actin (d) $(\times 20)$.

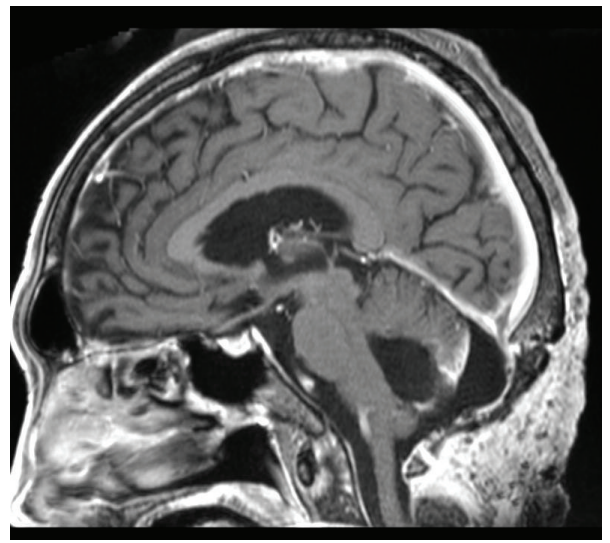

(a)

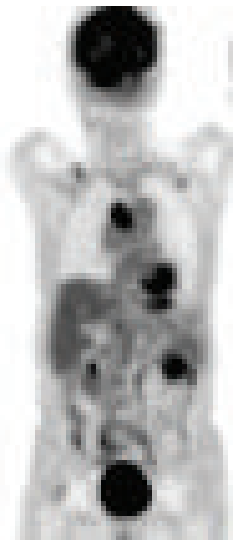

(b)

Figure 4: Postoperative MRI shows medulla decompressed without the residual tumor in the vermis (a). Positron emission tomography (PET) reveals abnormal accumulations in the cardiac apex, subclavial region, and peritoneal cavity (b).

(46\%) and peritoneum (41\%), while concurrent GIST and metastases to the central nervous system are extremely rare.

As shown in Table 1, only 16 cases (including the present) have been reported in the literature [8-22].

The majority of metastatic intracranial GISTs are present in older adults with a median age at presentation of approximately 60 years (mean, 55.7 years). Patients have sometimes been known to show metastatic disease elsewhere prior to the development of CNS metastases. The interval between treatment of the primary lesion and diagnosis of CNS metastasis is considerably long (14 months to 12 years; mean, 4.7 years), and the prognosis is dismal once intracranial metastasis is diagnosed. Mean overall survival has been documented as 8.6 months (range, 2-35 months). In the present case, cerebellar tumor was diagnosed based on immunohistochemical findings prior to diagnosis of the primary lesion. The primary 


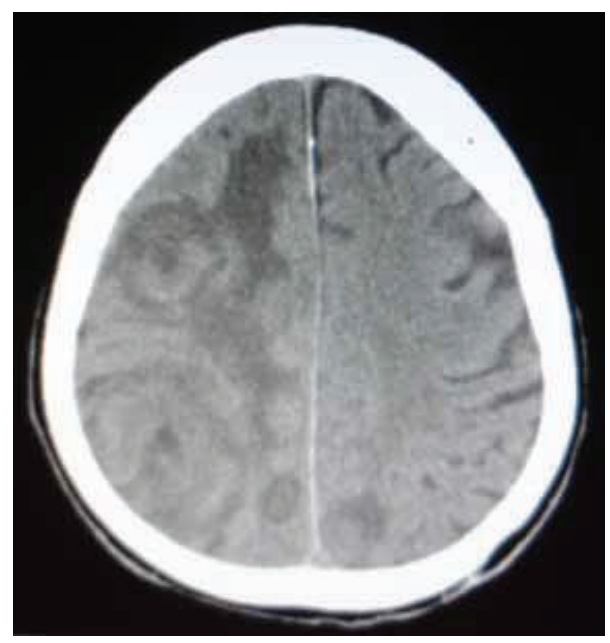

(a)

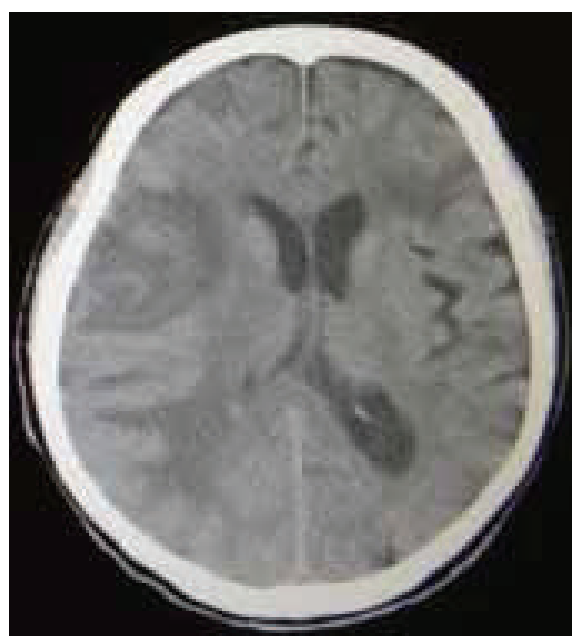

(b)

FIGURE 5: Following surgical operation and radiation therapy, computed tomography (CT) showed a large recurrent tumor with perifocal edema in the right frontal lobe (a) (b).

lesion turned out to be located in the small intestine and was identified when the patient suffered from ileus following craniotomy. In the three reported cases in which intracranial metastases were discovered first (including the present case), the prognosis was worse than that in those with intracranial metastases diagnosed after treatment of the primary lesion.

The current clinical practice guidelines recommend surgical resection for limited disease, particularly cortical or subcortical lesions, and adjuvant therapy as an option for patients with substantial risk of relapse [23].

Since tumor size $(>5 \mathrm{~cm})$ is one of the prognostic factors for aggressive behavior of GIST [24], the aim of surgery is to reduce the tumor volume to provide a cure and confirm the histological diagnosis if the metastatic tumor was discovered first, as in the present case. In such cases, surgical removal of the tumor is currently considered to be the first choice for the treatment of GIST, whether or not any distant metastases are present. Nine cases of intracranial metastasis were treated surgically. In eleven cases, patients underwent chemotherapy, including imatinib in nine patients and sunitinib in two patients. Nine patients were treated with radiation as an additional treatment. Only two of seven cases showed a response to chemotherapy. Seven patients achieved complete remission, and five of those seven patients had undergone surgery.

Recently, however, imatinib mesylate has been introduced as a molecular-targeted chemotherapeutic agent. Imatinib mesylate is an orally administered selective inhibitor of certain tyrosine kinases, including c-kit. Activating mutation of KIT or PDGFRA is found in the vast majority of GISTs, and the mutational status of these oncoproteins can be predictive of the clinical response to imatinib therapy $[2,5,25,26]$. The prognosis of GIST treated with imatinib mesylate has recently been reported. Fletcher et al. reported age, MIB1 index, and c-kit genetic mutation type as determining prognostic factors, whereas mutations in exon 11 of the c-kit gene correlate with the clinical outcomes of patients affected with GIST [24]. Although the genetic status of the primary lesion and CNS metastases is of interest, whether identical mutations were present in the primary and intracranial metastatic lesions remains unknown. Only five reports have described molecular features of metastatic intracranial GIST $[10,11,16,18,19]$, with only two patients showing KIT mutation (exon 9 and exon 11) $[10,16]$. Molecular findings of the primary and intracranial metastases were identical in both cases. The ability to identify the responsible gene in GISTs when KIT and PDGFRA are not mutated may lead to novel insights into the nature of GIST and new approaches to diagnosis and therapy.

Following resistance to imatinib mesylate, sunitinib, a multitargeted small-molecule tyrosine kinase inhibitor that selectively blocks vascular endothelial growth factor receptors (VEGFRs) with potent activity against KIT and PDGFA, has proven effective as a second-line therapy and is currently approved worldwide for metastatic GIST in patients with imatinib resistance or intolerance $[7,8]$. Unlike imatinib mesylate, sunitinib has the ability to penetrate the $\mathrm{BBB}[8,27$, 28 ], making this the treatment of choice when GIST is not amenable to surgery or the patient has uncontrolled lesions.

Although radiotherapy is not a standard therapy for GIST, Cuaron et al. described the importance of this option for the management of locally advanced and metastatic GISTs [29]. In a series of 18 patients treated with radiotherapy, the estimated 6-month local progression-free and overall survival rates were $57.0 \%$ and $57.8 \%$, respectively. For all patients, at least partial palliation was achieved in 17 patients (94.4\%), and symptoms were completely alleviated in eight patients (44\%). They concluded that radiotherapy can provide both objective responses and symptomatic relief without detracting dramatically from quality of life and should be considered in the multidisciplinary care of patients with locally advanced or metastatic GIST. 


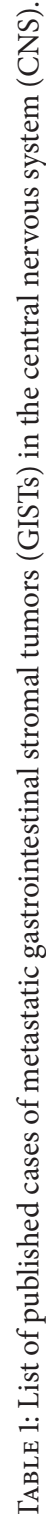

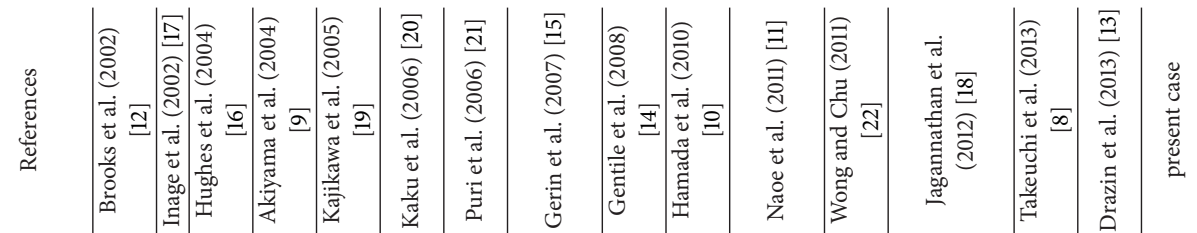

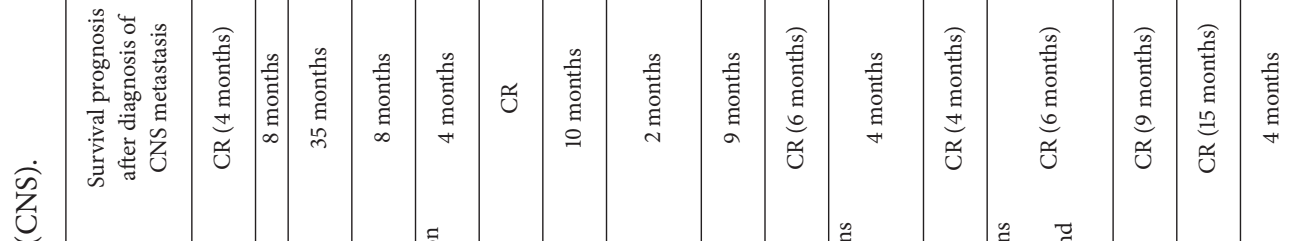

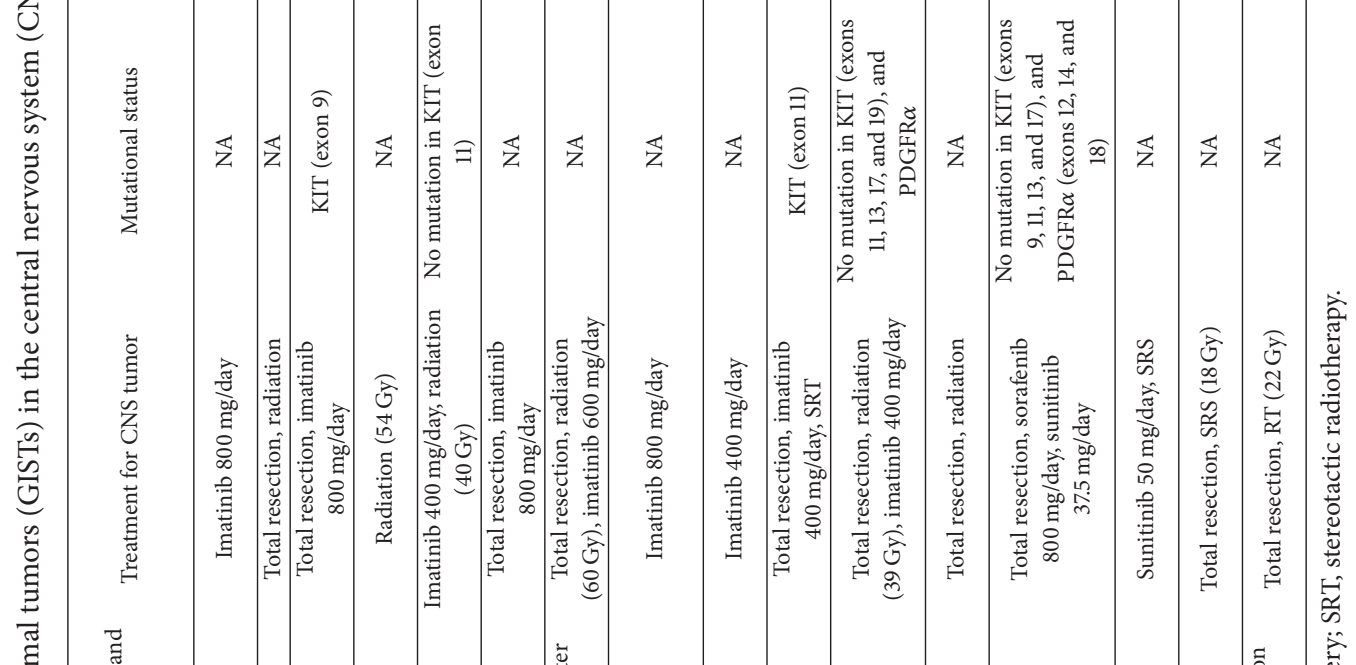

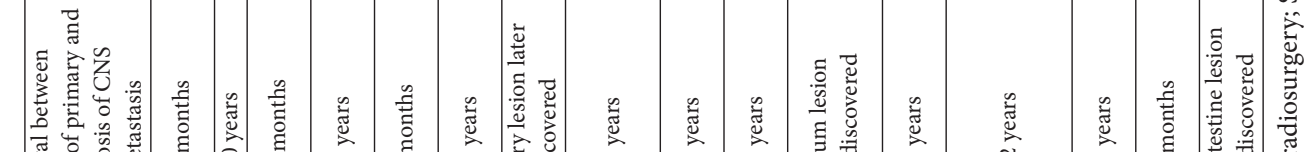

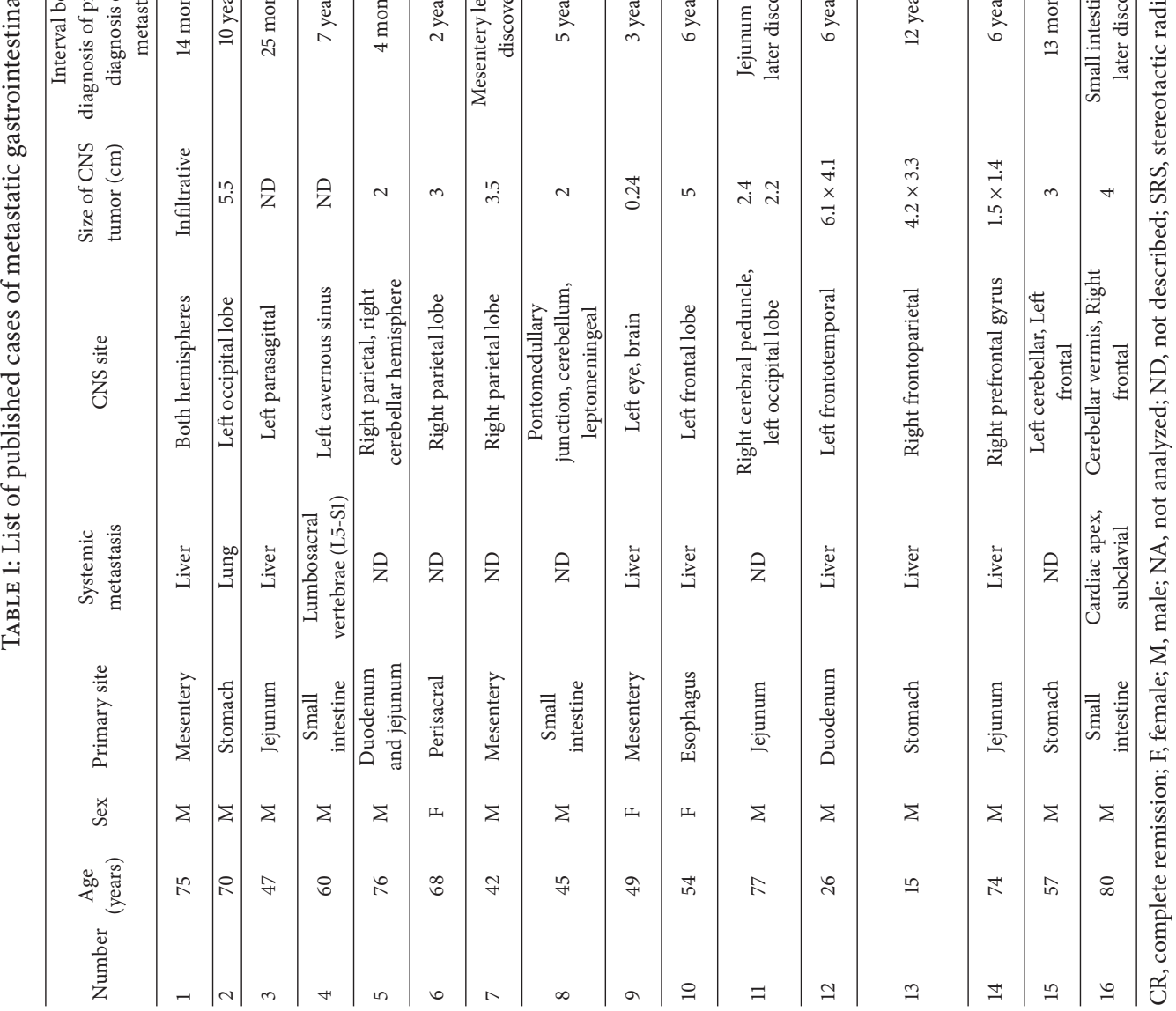


Despite the limitation of therapeutic strategies available, our findings remain hypothesis generating and offer important insights into a treatment modality that appears safe and effective for the management of patients with metastatic or locally advanced GIST.

\section{Conflict of Interests}

The authors declare that there is no conflict of interests regarding the publication of this paper.

\section{References}

[1] H. Chen, S. Hirota, K. Isozaki et al., "Polyclonal nature of diffuse proliferation of interstitial cells of Cajal in patients with familial and multiple gastrointestinal stromal tumours," Gut, vol. 51, no. 6, pp. 793-796, 2002.

[2] E. M. Connolly, E. Gaffney, and J. V. Reynolds, "Gastrointestinal stromal tumours," British Journal of Surgery, vol. 90, no. 10, pp. 1178-1186, 2003.

[3] M. Miettinen, M. Majidi, and J. Lasota, "Pathology and diagnostic criteria of gastrointestinal stromal tumors (GISTs): a review," European Journal of Cancer, vol. 38, no. 9, pp. S39-S51, 2002.

[4] G. D. Demetri, M. von Mehren, C. D. Blanke et al., "Efficacy and safety of imatinib mesylate in advanced gastrointestinal stromal tumors," The New England Journal of Medicine, vol. 347, no. 7, pp. 472-480, 2002.

[5] M. C. Heinrich, C. L. Corless, G. D. Demetri et al., "Kinase mutations and imatinib response in patients with metastatic gastrointestinal stromal tumor," Journal of Clinical Oncology, vol. 21, no. 23, pp. 4342-4349, 2003.

[6] A. L. Petzer, E. Gunsilius, M. Hayes et al., "Low concentrations of STI571 in the cerebrospinal fluid: a case report," British Journal of Haematology, vol. 117, no. 3, pp. 623-625, 2002.

[7] C. Serrano and S. George, "Recent advances in the treatment of gastrointestinal stromal tumors," Therapeutic Advances in Medical Oncology, vol. 6, no. 3, pp. 115-127, 2014.

[8] H. Takeuchi, H. Koike, T. Fujita, H. Tsujino, and Y. Iwamoto, "Sunitinib treatment for multiple brain metastases from jejunal gastrointestinal stromal tumor: case report," Neurol MedicoChirurgica, vol. 54, pp. 664-669, 2013.

[9] K. Akiyama, J. Numaga, F. Kagaya et al., "Case of optic nerve involvement in metastasis of a gastrointestinal stromal tumor," Japanese Journal of Ophthalmology, vol. 48, no. 2, pp. 166-168, 2004.

[10] S. Hamada, A. Itami, G. Watanabe et al., "Intracranial metastasis from an esophageal gastrointestinal stromal tumor," Internal Medicine, vol. 49, no. 8, pp. 781-785, 2010.

[11] H. Naoe, E. Kaku, Y. Ido et al., "Brain metastasis from gastrointestinal stromal tumor: a case report and review of the literature," Case Reports in Gastroenterology, vol. 5, no. 3, pp. 583-589, 2011.

[12] B. J. Brooks, J. C. Bani, C. D. M. Fletcher, and G. D. Demetri, "Challenges in oncology. Case 4. Response of metastatic gastrointestinal stromal tumor including CNS involvement to imatinib mesylate (STI-571)," Journal of Clinical Oncology, vol. 20, no. 3, pp. 870-872, 2002.

[13] D. Drazin, K. Spitler, S. Jeswani, A. Shirzadi, S. Bannykh, and C. Patil, "Multiple intracranial metastases from a gastric gastrointestinal stromal tumor," Journal of Clinical Neuroscience, vol. 20, no. 3, pp. 471-473, 2013.

[14] C. M. Gentile, A. A. Lombardi, and J. O. Croxatto, "Choroidal metastasis from gastrointestinal stromal tumour: a case report," British Journal of Ophthalmology, vol. 92, no. 1, pp. 156-157, 2008.

[15] F. Gerin, O. Baloglu, J. A. Morgan, and S. Kesari, "Central nervous system metastases from imatinib mesylate resistant gastrointestinal stromal tumor," Journal of Neuro-Oncology, vol. 82, no. 2, pp. 227-228, 2007.

[16] B. Hughes, D. Yip, D. Goldstein, P. Waring, V. Beshay, and G. Chong, "Cerebral relapse of metastatic gastrointestinal stromal tumor during treatment with imatinib mesylate: Case report," BMC Cancer, vol. 4, article 74, 2004.

[17] Y. Inage, K. Yamabe, T. Yamamoto et al., "Resection for pulmonary metastasis of gastrointestinal stromal tumor of the stomach at 10 years after gastrectomy; report of a case," The Japanese Journal of Thoracic Surgery, vol. 55, no. 10, pp. 907-911, 2002.

[18] J. P. Jagannathan, N. H. Ramaiya, A. B. Shinagare, J. L. Hornick, and S. George, "Intracranial metastasis from pediatric GI stromal tumor," Journal of Clinical Oncology, vol. 30, no. 10, pp. e122-e125, 2012.

[19] M. Kajikawa, A. Ishiyama, K. Sawada, K. Ono, and Y. Suzuki, "Multiple gastrointestinal stromal tumors of duodenum and jejunum accompanied by lymph node and brain metastasis: report of a case," Japanese Journal of Gastroenterological Surgery, vol. 38, no. 6, pp. 632-637, 2005.

[20] S. Kaku, T. Tanaka, T. Ohtuka et al., "Perisacral gastrointestinal stromal tumor with intracranial metastasis. Case report," Neurologia Medico-Chirurgica, vol. 46, no. 5, pp. 254-257, 2006.

[21] T. Puri, G. Gunabushanam, M. Malik et al., "Mesenteric gastrointestinal stromal tumour presenting as intracranial space occupying lesion," World Journal of Surgical Oncology, vol. 4, article 78, 2006.

[22] C.-S. Wong and Y.-C. Chu, "Intra-cranial metastasis of gastrointestinal stromal tumor," Chinese Medical Journal, vol. 124, no. 21, pp. 3595-3597, 2011.

[23] P. G. Casali and J.-Y. Blay, "Soft tissue sarcomas: ESMO clinical practice guidelines for diagnosis, treatment and follow-up," Annals of Oncology, vol. 21, supplement 5, pp. v198-v203, 2010.

[24] C. D. M. Fletcher, J. J. Berman, C. Corless et al., "Diagnosis of gastrointestinal stromal tumors: a consensus approach," Human Pathology, vol. 33, no. 5, pp. 459-465, 2002.

[25] C. L. Corless, J. A. Fletcher, and M. C. Heinrich, "Biology of gastrointestinal stromal tumors," Journal of Clinical Oncology, vol. 22, no. 18, pp. 3813-3825, 2004.

[26] S. Hirota, K. Isozaki, Y. Moriyama et al., "Gain-of-function mutations of c-kit in human gastrointestinal stromal tumors," Science, vol. 279, no. 5350, pp. 577-580, 1998.

[27] M. E. Gore, S. Hariharan, C. Porta et al., "Sunitinib in metastatic renal cell carcinoma patients with brain metastases," Cancer, vol. 117, no. 3, pp. 501-509, 2011.

[28] K. Senior, "Gleevec does not cross blood-brain barrier," Lancet Oncology, vol. 4, no. 4, p. 198, 2003.

[29] J. J. Cuaron, K. A. Goodman, N. Lee, and A. J. Wu, "External beam radiation therapy for locally advanced and metastatic gastrointestinal stromal tumors," Radiation Oncology, vol. 8, no. 1, article 274, 2013. 


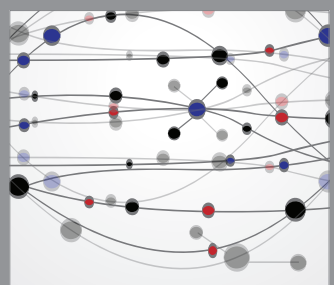

The Scientific World Journal
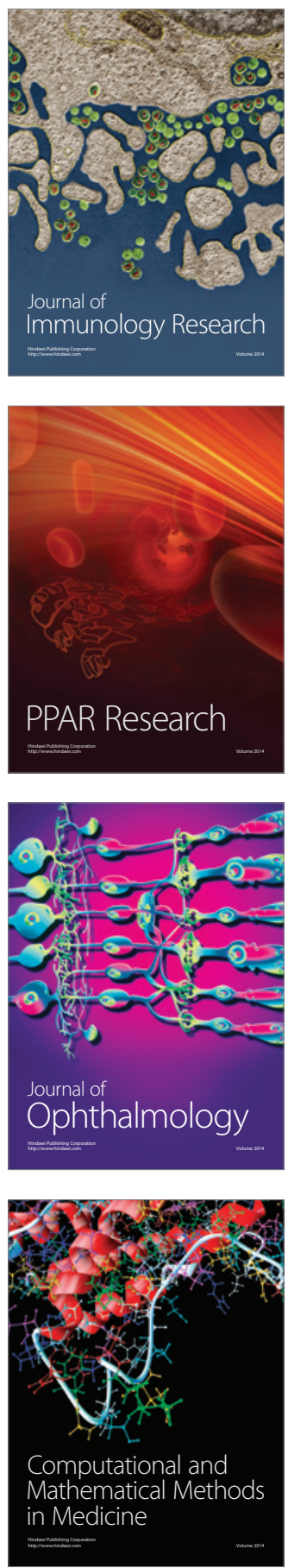

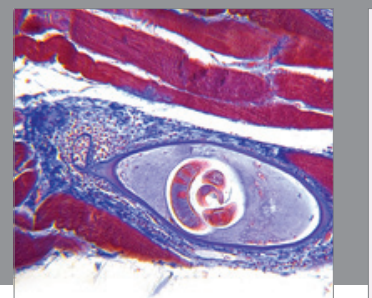

Gastroenterology

Research and Practice
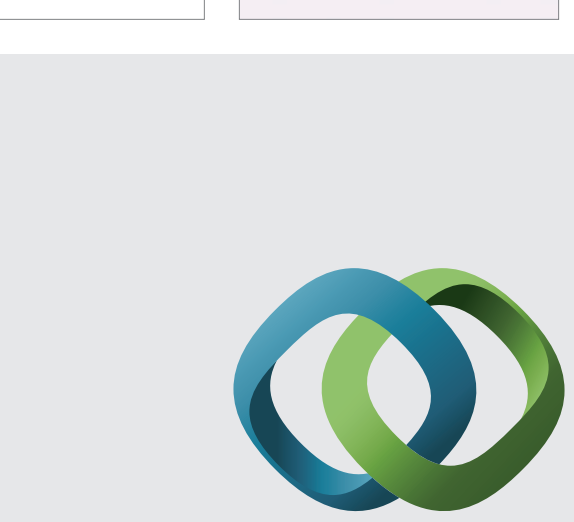

\section{Hindawi}

Submit your manuscripts at

http://www.hindawi.com
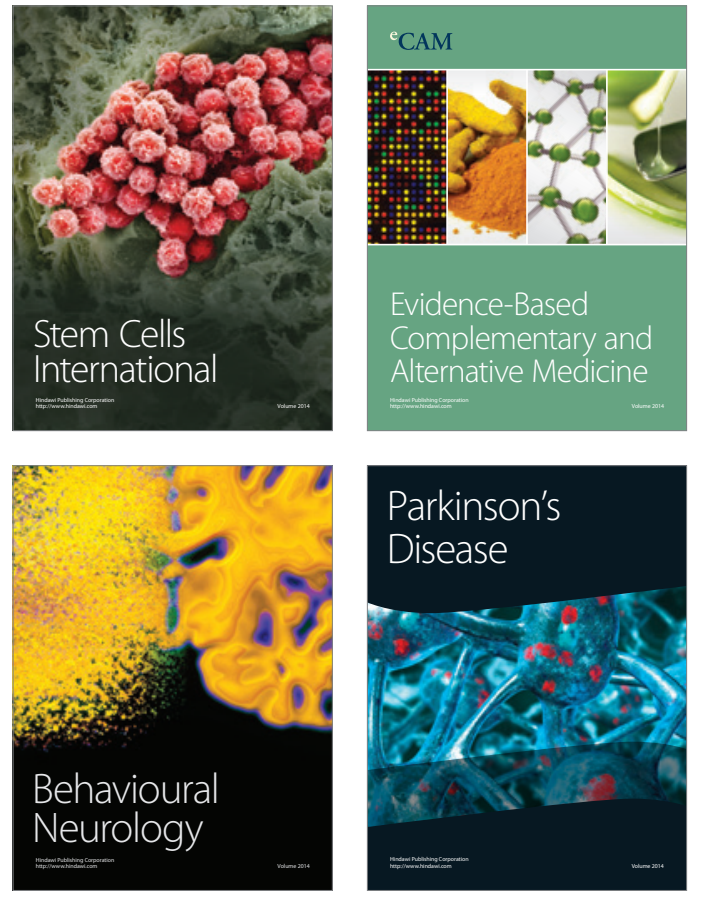
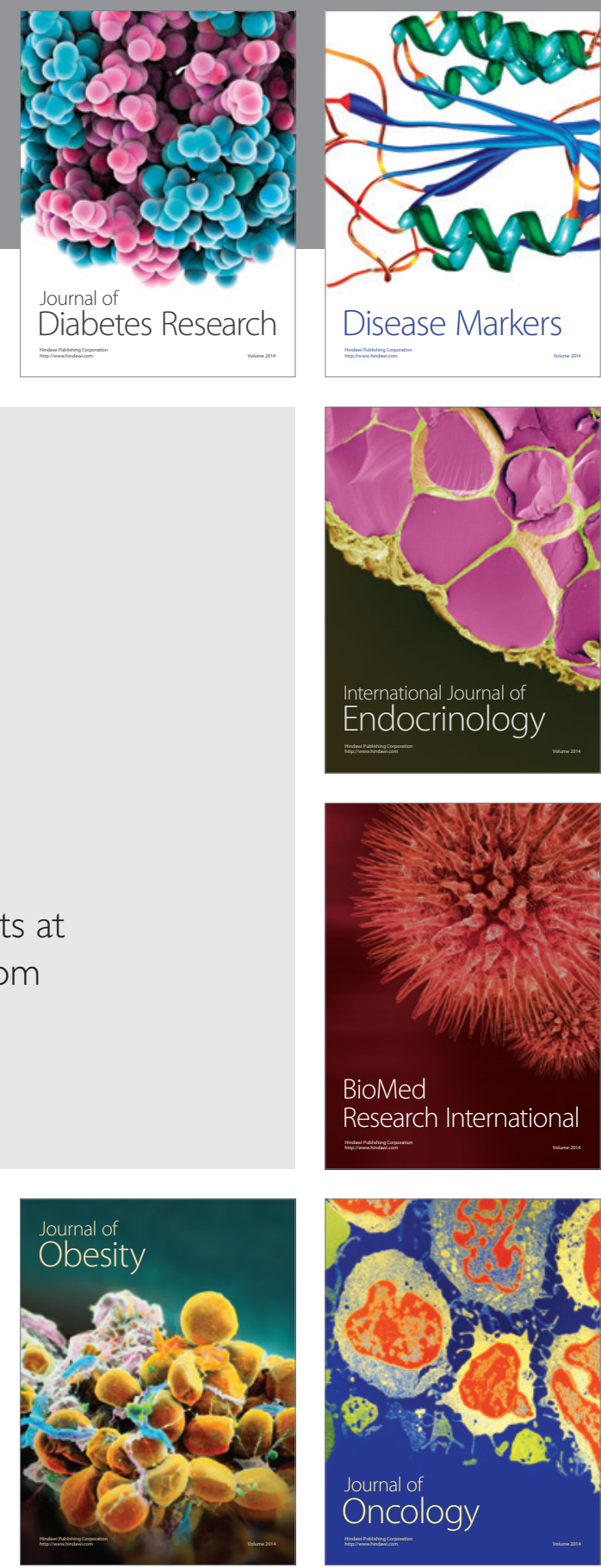

Disease Markers
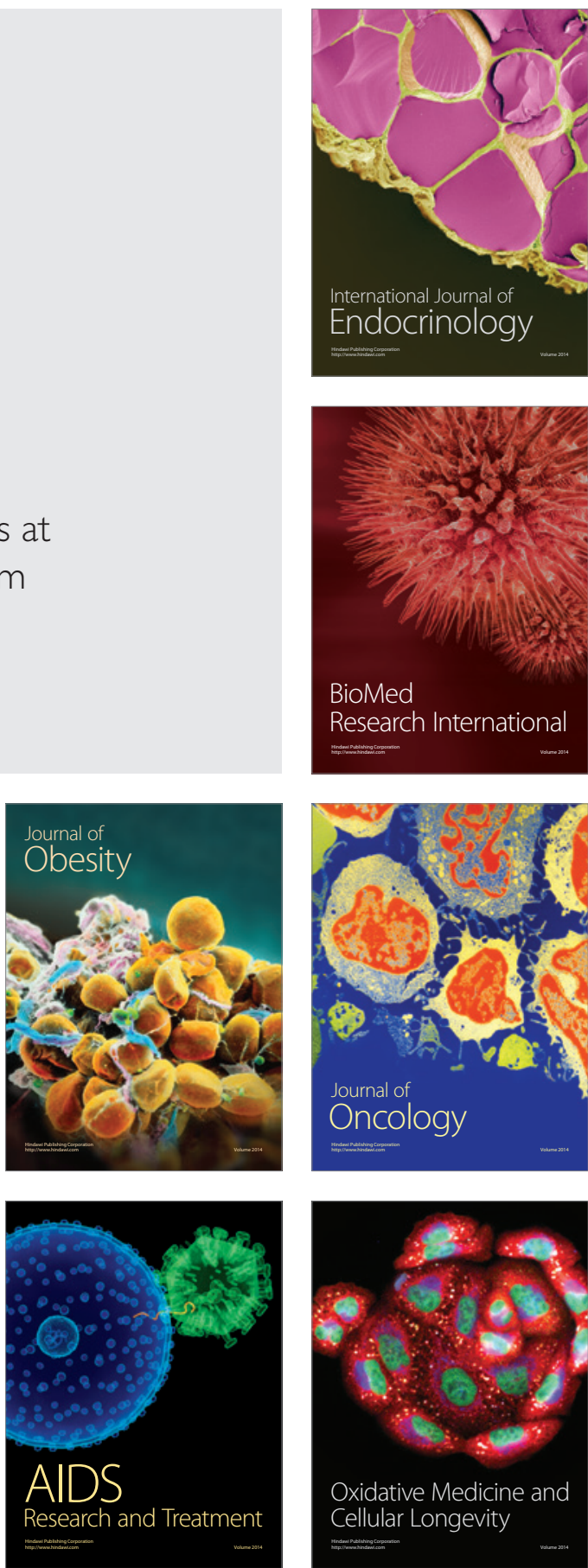